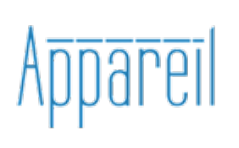

Appareil

$17 \mid 2016$

Art et médium 1 : le médium de l'art

\title{
La peinture moderniste
}

\section{Clement Greenberg}

Traducteur : Traduction de l'anglais (États-Unis) par Pascal Krajewski avec l'aimable autorisation de Sarah Greenberg Morse for the Estate of Clement Greenberg

\section{(2) OpenEdition \\ 1 Journals}

Édition électronique

URL : http://journals.openedition.org/appareil/2302

DOI : 10.4000/appareil.2302

ISSN : 2101-0714

Éditeur

MSH Paris Nord

Référence électronique

Clement Greenberg, "La peinture moderniste », Appareil [En ligne], 17 | 2016, mis en ligne le 12 juillet 2016, consulté le 30 juillet 2020. URL : http://journals.openedition.org/appareil/2302 ; DOI : https:// doi.org/10.4000/appareil.2302

Ce document a été généré automatiquement le 30 juillet 2020.

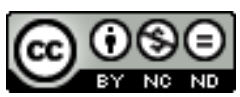

Appareil est mis à disposition selon les termes de la Licence Creative Commons Attribution - Pas d'Utilisation Commerciale - Pas de Modification 4.0 International 


\section{La peinture moderniste}

\section{Clement Greenberg}

Traduction : Traduction de l'anglais (États-Unis) par Pascal Krajewski avec l'aimable autorisation de Sarah Greenberg Morse for the Estate of Clement Greenberg

\section{La peinture moderniste}

1 Le Modernisme ne concerne pas que l'art et la littérature. Aujourd'hui, il touche presque l'intégralité de ce qui est véritablement vivant dans notre culture. Pour autant, il nous apparaît comme une véritable nouveauté historique. La civilisation occidentale n'est pas la première civilisation à inspecter et questionner ses propres fondements, mais elle est celle qui a été le plus loin dans ce sens. J'identifie l'avènement du Modernisme avec l'intensification, voire l'exacerbation de cette tendance auto-critique qui a commencé avec le philosophe Kant. Parce qu'il a été le premier à critiquer les moyens mêmes de la critique, je considère Kant comme le premier vrai moderniste.

2 L'essence du Modernisme, tel que je le vois, réside dans l'utilisation des méthodes spécifiques d'une discipline pour critiquer cette discipline elle-même, non pas afin de la subvertir mais dans le but de l'asseoir plus solidement dans son domaine de compétences. Kant a utilisé la logique pour établir les limites de la logique, et alors même qu'il ôtait beaucoup à l'ancienne juridiction de celle-ci, il la renforçait et l'assurait d'autant plus fermement dans son assise réduite.

3 L'auto-critique [self-criticism] du Modernisme fait son lit du criticisme des Lumières tout en s'en distinguant. Les Lumières critiquaient de l'extérieur, comme la critique au sens ordinaire du terme le fait habituellement; le Modernisme critique de l'intérieur, à travers les propres procédures de ce qui est critiqué. Il semble naturel que ce nouveau genre de critique soit d'abord apparu en philosophie, qui est critique par définition; mais plus le XVIII ${ }^{\mathrm{e}}$ siècle avançait, plus il pénétrait de nombreux autres domaines. On commença à exiger de toute forme d'activité sociale une justification plus rationnelle, et l'auto-criticisme kantien, qui avait d'abord émergé en philosophie pour répondre à cette exigence, finit par être convoqué dans des domaines bien éloignés de la philosophie, pour trouver et interpréter de telles justifications. 
4 Nous savons ce qui est arrivé à un domaine d'activités tel que la religion, qui ne pouvait pas recourir au criticisme kantien, immanent, pour se justifier. À première vue, on pourrait estimer que les arts se sont retrouvés dans une situation analogue à celle de la religion. S'étant vus refuser par la philosophie des Lumières toutes les tâches qu'ils auraient pu sérieusement faire leur, ils risquaient de se voir assimilés au divertissement pur et simple, et le divertissement lui-même semblait devoir être assimilé, au même titre que la religion, à la thérapie. Les arts ne pouvaient se sauver de ce nivellement par le bas qu'en démontrant que le genre d'expériences qu'ils offraient avait une valeur en soi et ne saurait être obtenu par un autre type d'activités.

5 Il s'avéra que chaque art devait produire cette démonstration pour son propre compte. Ce qui devait être exposé n'était pas seulement ce qui était unique et irréductible à l'art en général, mais aussi ce qui, pour chaque art particulier, lui était unique et irréductible. Chaque art devait déterminer, au travers de ses propres méthodes et œuvres, les effets qui lui étaient exclusifs. Ce faisant, il réduirait assurément son domaine de compétences, mais en même temps, il étayerait ce domaine de manière bien plus solide.

6 Il apparut rapidement que le domaine de compétences unique, propre à chaque art, coïncidait avec tout ce qui était unique dans la nature de son médium. La tâche de l'auto-critique consistait alors à éliminer, des effets spécifiques de chaque art, le moindre effet qui aurait éventuellement pu être emprunté à un autre médium, ou importé au travers du médium d'un autre art. Ainsi, chaque art serait rendu " pur » et trouverait dans sa "pureté » la garantie de son excellence ainsi que de son indépendance. « Pureté » signifiait auto-définition, et l'entreprise d'auto-critique dans les arts devint celle d'une auto-définition revancharde.

7 L'art réaliste ou naturaliste avait occulté le médium, en utilisant l'art pour se dissimuler comme art; le Modernisme utilisa son art pour attirer l'attention sur l'art. Les limites qui constituent le médium de la peinture - la surface plane, la forme du support, les propriétés du pigment - ont été traitées par les Maîtres Anciens comme des facteurs négatifs, qui ne se rencontraient qu'implicitement ou indirectement. Avec le Modernisme, ces mêmes limites furent considérées comme des facteurs positifs, et ont été ouvertement présentées. Les œuvres de Manet devinrent les premières peintures modernistes en vertu de la franchise avec laquelle elles affirmaient les surfaces planes sur lesquelles elles étaient peintes. Les impressionnistes, dans le sillage de Manet, abandonnèrent les sous-couches et les glacis, afin que l'œil n'ait aucun doute sur le fait que les couleurs qu'ils utilisaient provenaient de peinture en tubes ou en pots. Cézanne sacrifia la vraisemblance, ou l'exactitude, afin d'adapter plus explicitement ses traits et son dessin à la forme rectangulaire de la toile.

8 Et l'insistance sur l'inéluctable planéité de la surface [flatness of the surface] fut le point le plus fondamental dans les processus critiques par lesquels l'art de la peinture chercha à se définir au cours du Modernisme. Car la planéité était le seul élément exclusif de l'art pictural. Le format de la toile était certes une condition limitante, voire une norme, mais elle était partagée avec l'art du théâtre ; la couleur était une norme et un moyen que connaissaient non seulement le théâtre, mais aussi la sculpture. Parce que cette planéité était la seule condition que la peinture ne partageait avec aucun autre art, la peinture moderniste se tourna d'elle-même vers la planéité comme vers aucune autre chose. 
9 Les Maîtres Anciens avaient pressenti la nécessité de préserver ce qu'on appelle l'intégrité du plan de l'image [picture plane]: c'est-à-dire de signifier la présence durable de la planéité en-dessous et par-dessus l'illusion la plus éclatante d'un espace tridimensionnel. Le succès de leur art, comme de tout art pictural, tenait essentiellement à cette apparente contradiction. Les modernistes n'ont ni refusé ni résolu cette contradiction; bien plutôt, ils en ont inversé les termes. La planéité de leurs tableaux frappe l'observateur avant, et non plus après, la découverte du contenu de cette planéité. Alors que chez un Maître Ancien on a tendance à voir ce qui est peint avant de voir le tableau lui-même, on voit un tableau moderniste d'abord comme un tableau. Il s'agit là, bien sûr, de la meilleure façon de regarder tout tableau, ancien ou moderniste, mais le Modernisme l'impose comme la seule et nécessaire voie du regard, de sorte que le succès du Modernisme consacre le succès de l'auto-critique.

10 La peinture moderniste, en sa plus récente phase, n'a pas abandonné par principe la représentation d'objets reconnaissables. Ce qu'elle a abandonné par principe, c'est la représentation d'un certain type d'espace dans lequel des objets reconnaissables peuvent habiter. En soi, l'abstraction (ou la non-figuration) n'a toujours pas prouvé qu'elle était un moment absolument nécessaire de cette auto-critique de l'art pictural, même si des artistes aussi éminents que Kandinsky et Mondrian le pensaient. En soi, la représentation et l'illustration ne s'opposent pas à la spécificité de l'art pictural ; ce qui la contrarie, ce sont les liaisons entre les choses représentées. Toutes les choses reconnaissables (y compris les tableaux eux-mêmes) existent dans un espace tridimensionnel, et la moindre suggestion d'une chose reconnaissable suffit à convoquer les relations tridimensionnelles de ce genre d'espace. La silhouette fragmentaire d'une figure humaine, ou d'une tasse de thé, engendrera ce type d'espace relationnel, et, ce faisant, dénaturera l'espace pictural en lui ôtant sa stricte bidimensionnalité qui est pourtant la garantie de l'indépendance de la peinture comme art. Car, comme cela a déjà été dit, la tridimensionnalité est le royaume de la sculpture. Pour parvenir à l'autonomie, la peinture a surtout eu à se départir de tout ce qu'elle pouvait partager avec la sculpture, et c'est dans cet effort pour y parvenir - et pas tellement, je le répète, en excluant la représentation ou la fiction - que la peinture s'est faite abstraite.

11 Néanmoins, dans le même temps, la peinture moderniste démontre, justement par sa résistance au sculptural, à quel point elle reste fermement attachée à la tradition, en dépit des apparences. Car la résistance au sculptural est bien antérieure à la naissance $\mathrm{du}$ Modernisme. La peinture occidentale, jusqu'à son versant naturaliste, est grandement redevable à la sculpture, qui lui a enseigné dès le début comment porter les ombres et les modelés pour donner l'illusion du relief, et même comment user de cette illusion pour produire celle de la profondeur. Pourtant, certains des plus grands exploits de la peinture occidentale sont dus à l'effort qu'elle fit au cours des quatre derniers siècles pour se détacher de la sculpture. Naissant à Venise au XVI ${ }^{\mathrm{e}}$ siècle et se prolongeant en Espagne, en Belgique et en Hollande au XVII ${ }^{\mathrm{e}}$, cet effort fut d'abord accompli au nom de la couleur. Lorsque David, au XviII ${ }^{\mathrm{e}}$ siècle, a tenté de faire revivre la peinture sculpturale, c'était, en partie, pour sauver l'art pictural de l'aplatissement décoratif induit, semblait-il, par l'accent mis sur la couleur. Pourtant, la force des meilleurs tableaux de David, qui sont principalement ses toiles les moins travaillées, réside autant dans leurs couleurs que dans n'importe quel autre élément. Et Ingres, son fidèle élève, bien qu'il traitât la couleur comme secondaire, et ce avec une constance 
bien plus notable que David, exécuta des portraits qui ont été parmi les peintures les plus plates et les moins sculpturales faites en Occident depuis le XIV siècle par un artiste raffiné. Ainsi, au mitan du xix ${ }^{e}$ siècle, toutes les tendances ambitieuses de la peinture avaient fait converger leurs divergences vers un front anti-sculptural. Le Modernisme s'est inscrit dans cette tendance, en même temps qu'il la révélait à ellemême. Avec Manet et les impressionnistes, la question a cessé d'être celle de l'opposition entre la couleur et le dessin pour devenir celle de la séparation entre l'expérience purement optique et l'expérience optique altérée par des résonances tactiles. Ce fut au nom de l'optique pure et littérale, et non pas au nom de la couleur, que les impressionnistes prirent fait et cause contre les ombres, le modelage et tout ce qui, dans la peinture, pouvait connoter le sculptural. Ce fut, une fois encore, au nom du sculptural, avec ses ombres et ses modelés, que Cézanne, et après lui les cubistes, réagirent contre l'Impressionnisme, comme David avait réagi contre Fragonard. Mais là encore, tout comme la réaction de David et d'Ingres devait paradoxalement aboutir à une sorte de peinture encore moins sculpturale qu'auparavant, de même la contrerévolution cubiste s'abîma dans une sorte de peinture plus plate que tout ce que l'art occidental avait produit avant Giotto et Cimabue - et même si plate qu'elle pouvait à peine proposer des images reconnaissables.

Parallèlement, avec l'apparition du Modernisme, les autres règles cardinales de la peinture avaient commencé à se soumettre à une révision tout aussi fouillée, même si elle était moins spectaculaire. Il me faudrait plus de place que je n'en ai à ma disposition pour montrer comment la règle de la "forme contenant le tableau ", ou règle du cadre, fut assouplie, puis retendue, puis assouplie encore, et isolée, et ensuite retendue une fois de plus, par les générations successives des peintres modernistes. Ou bien comment les règles de finition et de texture de la peinture, ou encore celles de la valeur et du contraste des couleurs ont été révisées et révisées encore. De nouveaux risques furent pris au sujet de toutes ces règles, non seulement dans l'intérêt de l'expression, mais aussi afin de les exposer plus clairement comme règles. En les exhibant ainsi, c'est leur caractère indispensable que l'on testait. Ce test est loin d'être terminé, et le fait qu'il s'approfondisse à mesure qu'il se répète explique les simplifications autant que les complications radicales à l'œuvre dans la toute dernière peinture abstraite.

14 L'extrême n'est une question ni de caprice ni d'arbitraire. Au contraire, plus les règles d'une discipline sont définies étroitement, et moins elles sont susceptibles de laisser de liberté tous azimut. Les règles et les conventions essentielles de la peinture sont en même temps les conditions limitatives auxquelles un tableau doit se conformer pour être reçu comme tel. Le Modernisme a découvert que ces limites pouvaient être repoussées indéfiniment - avant qu'un tableau ne cesse d'être un tableau et ne se transforme en un objet arbitraire; mais il a également constaté que plus ces limites étaient repoussées plus elles devaient être respectées et indiquées explicitement. Les lignes noires entrecroisées et les rectangles de couleur d'une peinture de Mondrian semblent des éléments bien pauvres pour faire un tableau; mais ils imposent la forme du cadre du tableau comme norme régulatrice, avec une force et une unité sans précédent, en s'en faisant l'écho direct. Loin d'encourir le risque d'arbitraire, l'art de Mondrian se révèle avec le temps presque trop discipliné, et à certains égards presque trop lié à la tradition et à la convention. Une fois que nous nous sommes habitués à son abstraction totale, son art nous apparaît plutôt conservateur dans son usage de la 
couleur, par exemple, ou dans sa soumission au cadre, surtout comparé aux derniers tableaux de Monet.

Il va de soi, je l'espère, que pour dévoiler la rationalité de la peinture moderniste, j'ai dû simplifier et exagérer. La planéité vers laquelle la peinture moderniste se tourne ne pourra jamais être une planéité absolue. La sensibilité accrue au plan de l'image risque de ne plus permettre ni l'illusion sculpturale ni le trompe-l'œil, mais elle continue et doit continuer de permettre l'illusion optique. La première marque apposée sur une toile détruit sa planéité littérale et absolue, et le résultat des marques faites sur elle par un artiste comme Mondrian est encore une sorte d'illusion qui suggère un genre de troisième dimension. Mais ici, cette dernière est strictement picturale, strictement optique. Les Maîtres Anciens ont créé une illusion de l'espace en jouant sur la profondeur - et on pourrait s'imaginer y déambuler - mais l'illusion analogue créée par le peintre moderniste ne peut être vue, ne peut être traversée, littéralement ou au figuré, qu'avec les yeux.

La peinture abstraite la plus récente tente de reprendre à son compte l'insistance de l'Impressionnisme sur l'optique, seul sens que l'art pictural peut complètement et essentiellement invoquer. On peut aussi commencer dès lors à se rendre compte que les impressionnistes, ou au moins les néo-impressionnistes, ne faisaient pas forcément fausse route quand ils lorgnaient du côté des sciences. L'auto-criticisme kantien, tel qu'il nous apparaît aujourd'hui, a trouvé sa pleine expression dans la science plutôt que dans la philosophie, et quand on a commencé à l'appliquer en art, l'art s'est rapproché comme jamais de la méthode scientifique, et ce bien plus adéquatement qu'à la Renaissance avec Alberti, Uccello, Piero della Francesca, ou Léonard. Que l'art visuel doive se limiter exclusivement à ce qui est donné dans l'expérience visuelle, et qu'il ne doive faire aucun cas de ce qui peut être abordé par un autre régime d'expérience : c'est là un programme dont la seule justification réside dans une cohérence [consistency] d'ordre scientifique.

Seule la méthode scientifique exige, ou peut exiger, qu'une situation soit résolue exactement dans les mêmes termes que ceux dans lesquels elle a été posée. Mais ce genre de cohérence ne préjuge en rien de la qualité esthétique - même si l'art le plus intéressant de ces soixante-dix ou quatre-vingts dernières années s'est rapproché de plus en plus de cette cohérence. Du strict point de vue de l'art, sa convergence avec la science se trouve être un simple accident, et pas plus aujourd'hui qu'hier, l'art ni la science n'ont apporté à l'autre quelque surplus ou gage que ce soit. En revanche, ce que cette convergence entre l'art moderniste et la science moderne montre, c'est leur haut degré d'appartenance à une même tendance culturelle spécifique, ce qui est de la plus haute importance en tant que fait historique.

Il faut aussi comprendre que cette tendance à l'auto-critique de l'art moderniste n'a jamais été exploitée que d'une manière spontanée et largement subliminale. Comme je l'ai déjà indiqué, cela n'a toujours été qu'une question pratique, immanente à la pratique, et jamais un sujet théorique. On entend beaucoup parler des programmes concernant l'art moderniste, mais dans les faits, il fut bien moins question d'axe programmatique dans la peinture moderniste que dans celle de la Renaissance ou de l'Académie. À quelques exceptions près (comme Mondrian), les maîtres du Modernisme n'avaient pas plus d'idées arrêtées sur l'art que quelqu'un comme Corot. Certaines inclinations, certaines affirmations et accentuations, ainsi que certains refus ou abstentions s'imposent simplement d'eux-mêmes, parce que la voie vers un art plus 
fort et plus expressif se trouve à travers eux. Les objectifs immédiats des Modernistes étaient et restent avant tout individuels, de même que la vérité et la réussite de leurs œuvres sont singulières avant toute autre chose. Et seule l'accumulation d'une grande quantité de peinture personnelle a permis, au fil des décennies, de révéler la tendance auto-critique générale de la peinture moderniste. Aucun artiste n'était ou n'est encore conscient de cela; de même qu'aucun artiste ne peut jamais travailler librement en le sachant. Et de ce point de vue - et c'est là un fait non négligeable - l'art continue sur sa route avec le Modernisme comme il l'a toujours fait.

19 Et je ne saurais trop insister sur le fait que le Modernisme n'a jamais voulu signifier, hier pas plus qu'aujourd'hui, quelque chose comme une rupture avec le passé. On peut le voir comme une délitescence ou un détricotage de la tradition, mais il est tout aussi bien poursuite de son évolution. L'art moderniste continue le passé sans écart ni rupture, et quelle que soit sa forme terminale, il ne pourra être compris qu'en rapport avec le passé. Depuis ses débuts, la production de tableaux a été contrôlée par toutes les règles que j'ai mentionnées. Le peintre ou le graveur paléolithique purent ne pas tenir compte de la règle du cadre et traiter la surface d'une manière littéralement sculpturale, uniquement parce qu'ils faisaient des images plutôt que des tableaux, et parce qu'ils travaillaient sur un support - une paroi rocheuse, un os, une corne ou une pierre - dont les limites et la surface étaient arbitrairement fixées par la nature. Mais la réalisation de tableaux signifie, entre autres choses, l'assemblage ou le choix délibérés d'une surface plane, ainsi qu'un acte délibéré pour la circonscrire et la limiter. C'est précisément ce sur quoi la peinture moderniste joue: le fait que les conditions limitatives de l'art sont totalement et délibérément humaines.

Mais je tiens à répéter que l'art moderniste ne fournit pas de démonstrations théoriques. Au contraire, on pourrait dire qu'il lui arrive souvent de réaliser empiriquement des possibilités théoriques et, ce faisant, il teste la pertinence de nombreuses théories esthétiques à l'aune de la pratique et de l'expérience réelles de l'art. À cet égard, seul le Modernisme peut être considéré comme subversif. Certains facteurs que nous pensions essentiels au faire artistique et à l'expérience esthétique se sont révélés contingents quand la peinture moderniste a su se passer d'eux tout en continuant à offrir une pleine expérience de l'art. D'ailleurs, le fait que cette démonstration ait laissé la plupart de nos anciens jugements de valeur intacts, la rend encore plus concluante. Le Modernisme a peut-être eu quelque chose à voir avec le retour en grâce d'Uccello, Piero della Francesca, Le Greco, Georges de la Tour, et même Vermeer; et le Modernisme confirme, s'il ne l'a pas imposé, le regain d'intérêt pour Giotto; et pour autant, il n'a pas amoindri le statut des Léonard, Raphaël, Titien, Rubens, Rembrandt, ou Watteau. Ce que le Modernisme a montré, c'est que, si le passé a eu raison d'apprécier ces maitres, il l'a souvent fait pour des raisons mauvaises ou non pertinentes.

21 À certains égards, notre situation n'est pas meilleure aujourd'hui. La critique d'art et l'histoire de l'art sont en retard sur le Modernisme comme ils l'étaient sur l'art prémoderniste. La plupart des choses qui s'écrivent sur l'art moderniste relèvent encore du journalisme plutôt que de la critique ou de l'histoire de l'art. Il appartient au journalisme - et au système millénaire dont tant de journalistes et d'intellectuels souffrent de nos jours - de saluer chaque nouvelle phase de l'art moderniste comme le début d'une toute nouvelle époque dans l'art, marquant une rupture décisive avec toutes les habitudes et les conventions du passé. Chaque fois, on espère une nouvelle 
forme d'art, si différente des arts précédents, si affranchie des règles pratiques et des jugements de goût, que tout un chacun, quel que soit son niveau d'information, aurait voix au chapitre. Et à chaque fois, cette attente est déçue, car chaque nouvelle phase de l'art moderniste prend finalement place dans une histoire, continue et intelligible, du goût et de la tradition.

Rien ne pourrait être plus éloigné de l'art authentique de notre époque que l'idée d'une rupture de cette continuité. L'art est - entre autres choses - continu et il est impensable sans cela. S'il voulait se débarrasser de son passé, ou de son besoin compulsif à maintenir son niveau d'excellence, l'art moderniste manquerait autant de substance que de justification.

\section{Post-scriptum (1978)}

Le texte qui précède parut en 1960, en guise de pamphlet, dans une série publiée par Voice of America. Il fut aussi diffusé sur les ondes d'une antenne radio au printemps de la même année. Avec quelques modifications textuelles mineures, il fut réimprimé dans le numéro de printemps 1965 d'Art and Literature in Paris, puis dans l'anthologie de Gregory Battcock, The New Art (1966).

Je tiens à saisir cette occasion pour corriger une erreur, erreur d'interprétation et non de fait. Beaucoup de lecteurs, mais heureusement pas tous, semblent avoir compris la « rationalité » de l'art moderniste soulignée ici comme représentative d'une position adoptée par l'auteur lui-même, semblant croire que sa description valait plaidoyer. Il y avait là peut-être un défaut d'écriture ou rhétorique. Néanmoins, une lecture attentive de ce qui est écrit montre bien qu'en aucun cas l'auteur ne souscrit ou ne croit aux choses qu'il ne fait que discerner (les guillemets autour des mots " pure » et " pureté » auraient dû suffire à le montrer). L'auteur tente d'expliquer partiellement les processus par lesquels est apparue la plus grande partie du meilleur art de ce dernier siècle, mais il ne veut pas dire par là qu'elle devait nécessairement apparaître ainsi, ni que le meilleur de l'art futur suivra cette voie. L'art « pur » était une illusion utile, mais il n'en reste pas moins une illusion. Qu'il puisse continuer d'être utile ne le dédouane pas davantage d'être une illusion.

Il y a eu d'autres utilisations de ce que j'ai écrit qui sont devenues absurdes à force d'être outrées : celle qui affirme que je considère la planéité et l'astreinte à la planéité non seulement comme les conditions limitatives de l'art pictural, mais aussi comme les critères de qualité esthétique de l'art pictural; ou celle soutenant que plus loin s'aventure l'œuvre sur la voie de l'auto-définition de son art, meilleure elle est. Le philosophe ou l'historien de l'art - ou qui que ce soit - qui peut croire un instant me voir ainsi aboutir à des jugements esthétiques, fait là une scandaleuse lecture de luimême plutôt que de mon article. 


\section{RÉSUMÉS}

"Modernist painting" est paru en 1960 dans Voice of America, soit vingt ans après "Towards a newer Laocoon". Greenberg y revient sur son interprétation historique de la peinture moderniste et son analyse de la "pureté ». Il définit le Modernisme comme la tendance à l'auto-critique, tendance que l'on peut trouver à l'œuvre dans tout domaine d'activités et qui est issue, grosso modo, de Kant. En art, une telle auto-critique passe par une interrogation sur la nature du médium de chaque art. Ainsi peut s'élaborer une auto-définition de chaque art, ramené à sa pureté, c'est-à-dire à ce qu'il ne partage avec aucun autre art. En peinture, cet élément, ce propre, consiste en la planéité de la surface de la toile; la peinture moderniste, formelle y trouvera donc son axe exclusif d'exploration.

Dès lors, voir un tableau moderniste consiste à voir d'abord et avant tout le tableau, plutôt que ce qui y est peint. La peinture moderniste va être amenée à refuser un certain type d'espace, représentatif d'une profondeur réaliste et donc contraire à la stricte bidimensionnalité de la toile. Elle s'oppose alors à la sculpture et se rattache donc à une longue tradition antisculpturale : celle-ci a commencé quand la peinture insistait sur la couleur, puis continua au XIX siècle, alors qu'elle mettait l'accent sur l'optique pure. Cette histoire de la peinture est dialectique, faite de révolutions et de contre-révolutions qui conduisent jusqu'à l'affirmation de la planéité du tableau. Cette démarche auto-critique révèle encore l'usage fécond des règles limitatives pour créer, et l'inspiration des méthodes scientifiques pour se donner une cohérence d'ordre scientifique. Enfin Greenberg revient sur une idée fausse, soutenant qu'il n'y a pas de rupture avec la peinture du passé.

\section{INDEX}

Mots-clés : modernisme, autocritique, peinture, planéité, histoire, tradition 